\title{
MOOCs e Colaboração: definição, desafios, tendências e perspectivas
}

\author{
Ana Carla A. Holanda ${ }^{1}$, Patrícia C. A. Restelli Tedesco ${ }^{1}$ \\ ${ }^{1}$ Universidade Federal de Pernambuco (UFPE) \\ Caixa Postal 50.540-740 - Recife - PE - Brasil \\ \{acah@cin.ufpe.br, pcart@cin.ufpe.br\}
}

\begin{abstract}
Mass Online Open Courses (MOOCs) are educational environments made available through the Web and have the characteristic of dealing with a large number of students and should constantly stimulate the participation and collaboration of those involved. This article presents the results of a Systematic Literature Review (RSL), carried out to investigate which resources are used by MOOCs to guarantee collaborative environments. We selected 187 scientific articles published until January 2017 that were evaluated according to previously defined inclusion and exclusion criteria. Among the results, it can be highlighted that the difficulty in mapping student profiles and context replication can be minimized through Data Mining and Learning Analitycs. Thus, from the RSL, it was observed that although scholars are aware of the need to encourage collaboration, few actions are effectively implemented in MOOC environments.
\end{abstract}

\section{Resumo}

Os Cursos Abertos Massivos Online (MOOCs) são ambientes educacionais disponibilizados por meio da Web e possuem a característica de lidar com um grande número de estudantes e devem estimular constantemente a participação e a colaboração dos envolvidos. Este artigo apresenta os resultados de uma Revisão Sistemática da Literatura (RSL) executada para investigar quais recursos são utilizados pelos MOOCs para garantir ambientes colaborativos. Foram selecionados 187 artigos científicos publicados até janeiro 2017 que foram avaliados de acordo com critérios de inclusão e exclusão previamente definidos. Dentre os resultados, pode-se destacar que a dificuldade no mapeamento de perfis dos alunos e a replicação do contexto pode ser minimizada através de Mineração de Dados e Learning Analitycs. Assim, a partir da RSL, observou-se que apesar dos estudiosos terem consciência da necessidade de incentivar a colaboração, poucas ações são implementadas de forma efetiva em ambientes MOOCs.

\section{Introdução}

MOOC tem chamado a atenção dos estudiosos da educação como uma nova possibilidade de acesso à aprendizagem. A proposta de uma forma geral é servirem como plataformas de conhecimento para qualquer um, a qualquer hora, e em qualquer lugar, fazendo deles 
VI Congresso Brasileiro de Informática na Educação (CBIE 2017)

Anais do XXVIII Simpósio Brasileiro de Informática na Educação (SBIE 2017)

uma emergente e poderosa estratégia de aprendizagem com repercussão nas áreas tecnológica e educacional segundo Zheng et al. (2016).

Entretanto, existem dúvidas quanto à efetividade dos cursos MOOCs, tendo em vista a suas características. De acordo Chauan et al. (2015), as pesquisas demonstram que muitos são os desafios para disponibilizar cursos que promovam a aprendizagem colaborativa. A colaboração é cada vez mais necessária nos contextos acadêmicos atuais e na vida profissional.

Embora a colaboração tenha o potencial para promover a aprendizagem, não é fácil consegui-la. Para conduzir com sucesso uma atividade de aprendizagem colaborativa são necessárias várias condições, por exemplo: i) a existência de um objetivo comum (Nelimarkka and Vihavainen 2015); ii) a interdependência positiva entre pares (Nelimarkka and Vihavainen 2015); iii) os mecanismos de coordenação e comunicação (Zheng et al. 2016); iv) a responsabilidade individual (Chauan et al. 2015), entre outros.

Dessa forma, esta pesquisa teve como objetivo avaliar o contexto atual dos MOOCs a partir de uma Revisão Sistemática da Literatura (RSL) para que fosse possível entender as suas caracterizações, suas particularidades, bem como os desafios e propostas de implementação encontrados para que se possa nortear as futuras pesquisas relacionadas ao assunto.

O artigo está organizado da seguinte forma: a Seção 2 apresenta a metodologia de Revisão Sistemática conduzida no contexto de definir MOOCs, verificar a relação necessária com ambientes colaborativos, bem como compreender os desafios que garantam cursos colaborativos e que promovam aprendizagem crítica e criativa. Na Seção 3 são discutidas as evidências encontradas como respostas às questões de pesquisa definidas no protocolo da RSL. Por fim, na Seção 4 são apresentadas as conclusões e perspectivas para a continuidade do trabalho.

\section{Visão Geral da Revisão Sistemática}

De acordo com Kitchenham (2004), uma Revisão Sistemática da Literatura (RSL) é uma técnica de pesquisa que identifica, avalia e interpreta todas as pesquisas relevantes disponíveis para uma determinada questão de pesquisa seguindo as fases de um protocolo bem definido. Através de uma RSL podemos avaliar as pesquisas realizadas tendo como base uma determinada área ou tópico de pesquisa visando identificar eventuais lacunas, a fim de sugerir áreas para investigação. Também pode ser utilizada para ajudar na geração de novas hipóteses de pesquisa a partir de background conseguido com a revisão.

Para realizar uma RSL é necessário seguir alguns passos:

1 - Planejamento - nesta etapa foi definido um protocolo de pesquisa para evitar resultados errôneos ou tendenciosos. Para isso ser possível foi necessário definir as questões primárias de pesquisa que nortearam a realização do trabalho. As questões foram definidas a seguir:

Q1. Como os MOOCs são definidos e qual a sua classificação?

Q2. Quais os desafios encontrados na construção e condução de MOOCs? 
VI Congresso Brasileiro de Informática na Educação (CBIE 2017)

Anais do XXVIII Simpósio Brasileiro de Informática na Educação (SBIE 2017)

\section{Q3. Que ferramentas colaborativas são utilizadas em ambientes MOOCs? \\ Q4. Como a colaboração está sendo implementada nos ambientes MOOC?}

As buscas de artigos foram realizadas por meio da submissão de strings às bases de dados indexadas e máquina de busca eletrônica, conforme destacado na Tabela 1. A string geral de busca foi pensada tentando relacionar aspectos de ambientes MOOCs e meios de colaboração, conforme definida abaixo:

Tabela 1: string de busca da RSL

(mooc OR massive open online course) AND (collaborative learning OR collaboration OR collaborative tool OR collaborative media)

Foram utilizados os seguintes critérios de inclusão a fim de eliminar estudos irrelevantes para o objeto de pesquisa, conforme mostrado abaixo:

CI.1. O artigo ajuda a definir e classificar MOOCs.

CI.2. O artigo discute pesquisas específicas e aplicadas em MOOCs, tais como sistemas de recomendação, personalização, adaptação, colaboração.

CI.3. O artigo relata experiências relacionadas a aspectos de colaboração.

Além desses critérios também foram adotadas as seguintes restrições: artigos duplicados, de opinião, sem a versão completa e artigos que não foram publicados em periódicos ou anais de conferências.

2 - Execução - após o planejamento foram realizadas as buscas levando em consideração o protocolo de pesquisa definido na etapa anterior. Foram encontradas 187 publicações científicas. Para selecionar os artigos foram lidos o título, resumo e palavras-chave de todos os artigos. Das 187 publicações foram lidos na íntegra 69 artigos selecionados após a avaliação baseado nos critérios de inclusão identificados acima. A Figura 1, mostra a evolução da seleção dos artigos. A lista de artigos pode ser visualizada em https://www.dropbox.com/s/8hj4x8g0xo88h08/Planilha\%20artigos.xlsx?dl=0

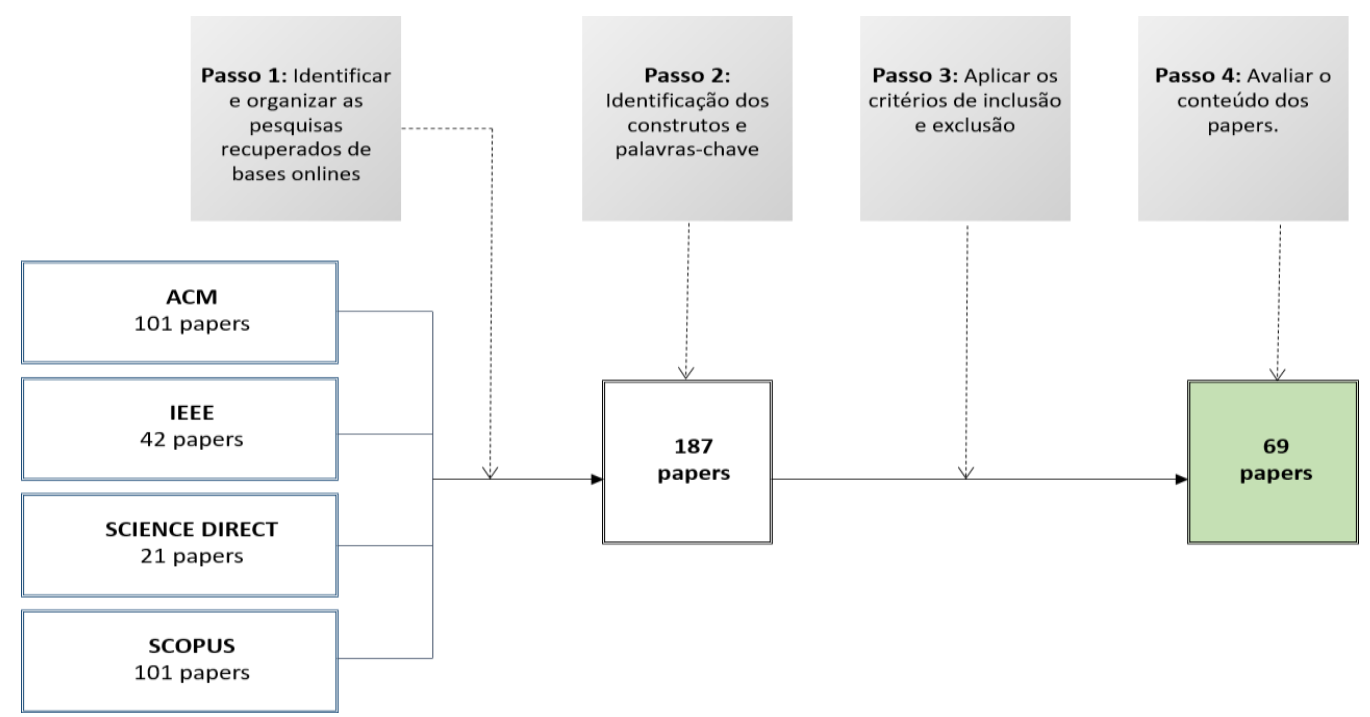

Figura 1: Evolução seleção dos artigos 
VI Congresso Brasileiro de Informática na Educação (CBIE 2017)

Anais do XXVIII Simpósio Brasileiro de Informática na Educação (SBIE 2017)

3 - Síntese da revisão - Neste momento foram realizadas as análises dos achados encontrados com a RSL e discussões foram necessárias para sugerir propostas de novas investigações no que se refere a MOOCs e ambientes colaborativos.

\section{Análise e Discussão dos dados}

A partir da RSL foi possível responder as questões de pesquisa definidas na etapa de planejamento a partir do protocolo de pesquisa.

\section{Q1 - Como os MOOCs são definidos e qual a sua classificação?}

Cursos Massivos Abertos Online (MOOC) tem tido um rápido crescimento e atraído a atenção de várias instituições como uma alternativa para disponibilizar qualificação e aprendizagem, seja através de cursos isolados ou através de cursos superiores, para uma ampla quantidade de pessoas através da Internet. Contudo, apesar da aceitação generalizada dos MOOCs como uma oportunidade para transformar as práticas educacionais, permanecem muitas questões quanto à sua sustentabilidade, as altas taxas de desistência e os desafios relacionados com a aprendizagem, baseado em Zheng et al. (2016). Além disso, pode-se acrescentar aspectos colaborativos e de interação nesses ambientes.

Nesse sentido, se faz necessário definir MOOCs para que haja uma maior compreensão do tema em questão. De acordo com a RSL proposta, MOOC pode ser definido como sendo um curso disponibilizado por meio da Internet, sem custo, oferecido para um número muito grande de pessoas, como comenta Chauan et al. (2015). Para Gené et al. (2014]), MOOC é um curso gratuito, baseado na web, com o registro aberto e currículo compartilhado publicamente. Já Siemens (2013) sustenta que MOOCs são uma continuação da tendência em inovação, experimentação e do uso da tecnologia iniciada pelo ensino a distância e online, para oferecer oportunidades de aprendizagem de forma massiva.

Também de acordo com Oliveira (2013) pode-se definir MOOCs como um ambiente educacional disponibilizado por meio da Web (através de AVA e/ou ferramentas da Web 2.0 e/ou Redes Sociais) que tem como principal objetivo disponibilizar para um grande número de alunos a oportunidade de acesso a novos conhecimentos e objetos de aprendizagem.

Existem muitas dúvidas quanto à diferença dos cursos on-line tradicionais e MOOCs. Neste sentido, estes diferem-se daqueles pela quantidade de alunos que podem participar dos cursos. A massividade é um dos principais diferenciais dos cursos MOOC. De acordo com Dasarathy et al. (2014), "massivo" define-se por ter mais registrantes do que assistentes e professores capazes de interagir e se comunicar. Já Coetzee et al. (2014), por sua vez, em contraste com cursos à distância tradicionais, diz que "massivo" se refere a um número ilimitado de participantes.

Além disso, de acordo com Leríz et al. (2016) os estudantes auto-organizam sua participação de acordo com as suas metas de aprendizagem, conhecimento prévio e as habilidades e interesses. Embora possa compartilhar algumas das convenções de um curso 
VI Congresso Brasileiro de Informática na Educação (CBIE 2017)

Anais do XXVIII Simpósio Brasileiro de Informática na Educação (SBIE 2017)

normal, como um cronograma pré-definido e temas semanais para apreciação, em um MOOC geralmente não há expectativas pré-definidas para a participação e geralmente não fornecem acreditação formal.

É importante destacar que nem todos os cursos oferecidos sob o nome MOOC são iniciados e guiados pelas mesmas concepções pedagógicas, baseado em Siemens (2013). Assim, se faz necessário classificar os tipos de MOOCs para melhor entender a sua condução no processo de aprendizagem.

Em relação à classificação dos MOOCs, Siemens (2013) diz que existe uma nítida convergência em classificá-los em xMOOCs e cMOOCs, conforme abaixo:

- cMOOCs (Connectivism-based MOOCs): seguem ideias da teoria conectivista e cursos baseados em networking. Enfatizam a aprendizagem colaborativa e compartilham a noção de participação livre em um curso sem crédito. Enfatizam a aprendizagem colaborativa conectada e os cursos são construídos em torno de um grupo de "indivíduos" de mentalidade semelhante, relativamente livres de constrangimentos institucionais como comenta Zheng et al. (2016).

- xMOOCs: são extensões de cursos online convencionais. Seguem uma abordagem Behaviorista, de acordo com Blanco et al. (2013). É essencialmente uma extensão dos modelos pedagógicos praticados dentro das próprias instituições. Em essência, são consideradas como instrução tradicional, centrada no professor, enriquecida em tecnologia. Tais sistemas oferecem uma experiência individualizada em que permitem que os estudantes façam exame de rotas alternativas através do material e ofereçam o gabarito automatizado. No entanto, eles não fornecem uma experiência de aprendizagem social ou de ser tratado pessoalmente. Tem seu foco no conteúdo e são centralizados em uma plataforma que utiliza ferramentas de classificação automatizada para apoiar milhares de alunos. As empresas e parceiras que se enquadram incluem Coursera, SEE, MIT, Harvard, EdX, Udacity, entre outras.

\section{Q2. Quais os desafios encontrados na construção e condução de MOOCs?}

A construção e o uso de MOOCs envolve vários desafios devido ao grande número de alunos (talvez na ordem de vários milhares) e seus backgrounds heterogêneos como afirma Nelimarkka et al. (2015). Estes desafios incluem o desenvolvimento e integração de materiais ou ferramentas abertas, a concepção de infraestrutura de um sistema adaptável e suporte a atividades. Outro desafio é a forma de captura e acompanhamento da interação dos alunos com o objetivo de mensurar e caracterizar a experiência de aprendizagem de sucesso e replicar para outros contextos.

Também pode ser citado a dificuldade em mapear os perfis dos alunos baseado em seu comportamento e interação. Segundo Chauhan et al. (2015), essa personalização podem aumentar a motivação dos alunos em MOOCs. Em consequência, um MOOC requer um modelo pedagógico flexível baseado em uma alta interação e automotivação. Nesse sentido, os modernos métodos educacionais sugerem que os alunos devem desenvolver habilidades como: criatividade, pensamento crítico, colaboração e resolução 
VI Congresso Brasileiro de Informática na Educação (CBIE 2017)

Anais do XXVIII Simpósio Brasileiro de Informática na Educação (SBIE 2017)

de problemas, com o objetivo de enfrentar desafios do século XXI baseado em Dasarathy et al. (2014).

O acompanhamento da interação dos alunos durante o curso foi citado por Léris et al. (2016). Segundo o artigo, a forma como ocorre esse acompanhamento é fundamental para identificar e planejar objetivos das atividades, meios e canais de comunicação, sistemas de recompensa, entre outras estratégias para promover maior interação e colaboração. Léris et al. (2016) afirma ainda que o modo de avaliação é outro desafio detectado, tendo em vista que é necessário definir uma estratégia de avaliação para medir o nível de aprendizagem adquirido durante a atividade colaborativa. Entender e analisar o processo de aprendizagem colaborativa requer uma análise baseada na interação dos alunos baseados nos contextos de aprendizagem.

A evasão em cursos MOOC é um desafio preocupante, pois mesmo como o crescimento dos MOOCS aceito como uma oportunidade única para transformar as práticas educacionais, permanecem muitas questões quanto à sua sustentabilidade, devido às altas taxas de desistência, como proposto por Zheng et al. (2016). Várias pesquisas têm sido realizadas com o intuito de detectar formas de diminuir a evasão. Além disso, os autores também abordam o fato de que a construção de ambientes MOOCs na perspectiva somente do aluno traz dificuldades de interação e até evasão, tendo em vista que o professor fica descontextualizado do processo de aprendizagem como agente ativo e envolvido no processo de aprendizagem.

De acordo com Lerís et al. (2016) uma interação social entre os alunos promove o entendimento uns dos outros através de apoio, ajuda e participação nas atividades de aprendizagem. Já Oliveira (2013) complementa afirmando que a construção de um contexto social pode motivar os alunos com seus colegas ou instrutores a compartilhar informações e aprender para trabalhar como um grupo. Além disso, através dos serviços de interação social, a motivação dos alunos para aprender, desde que guiada por uma estratégia colaborativa, é aumentada. Para sintetizar os achados encontrados na RSL, a Figura 3, abaixo mostra a média de ocorrência dos desafios encontrados nos artigos.

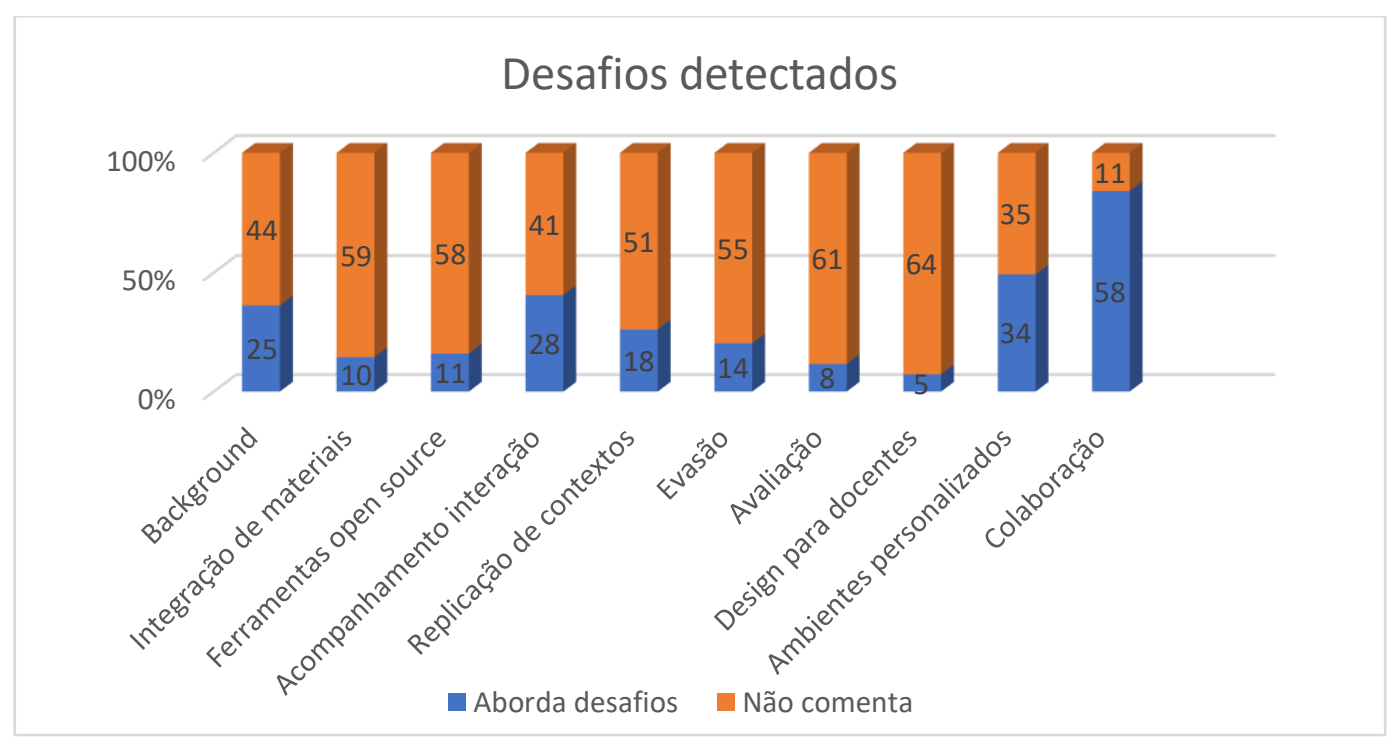

Figura 2: Desafios detectados com a utilização dos MOOCs 
VI Congresso Brasileiro de Informática na Educação (CBIE 2017)

Anais do XXVIII Simpósio Brasileiro de Informática na Educação (SBIE 2017)

\section{Q3. Que ferramentas colaborativas são utilizadas em ambientes MOOCs?}

Para Santos et al. (2012), um Sistema Colaborativo se constitui num ciberespaço, que é o espaço de convivência da nova sociedade em rede, um espaço para as interações humanas que possibilita vivenciar experiências intensas e tem grande poder de atrair e manter frequentadores. Assim, é necessário criar espaços para serem habitados e possibilitar novas formas de trabalho e de interação social. Para criar esses espaços citados acima são necessárias ferramentas ou ambientes que possibilitem colaboração.

Percebe-se que colaboração é uma característica notória e relevante para promover aprendizagem em MOOCs e diversas pesquisas têm sido realizadas neste sentido. Dentre as ferramentas algumas chamam atenção. Vários estudos mostram que mecanismos assíncronos são mais eficazes quando combinados com mecanismos síncronos, como salas de bate-papo em tempo real (Chat) e mensagens privadas, como afirma Coetzee (2014). As pesquisas têm investigado o papel dos chats em contextos de trabalho, entretenimento e em contextos educacionais.

Para Gené et al. (2014) outra ferramenta encontrada nas pesquisas são os jogos. A incorporação de metodologias de gamificação podem aumentar a motivação de participantes através do uso de mecanismos de jogo e influencia maior participação, compromisso e lealdade dos alunos podendo gerar um número maior de participantes proativos.

Técnicas de personalização são uma solução clássica recomendada por muitos especialistas para melhorar o aprendizado. As tecnologias da informação e da comunicação ajudaram professores a enfrentar uma diversidade de perfis de estudantes e um grande número de alunos em uma sala de aula. Quando esses fatores são extremos, como em MOOC, essas técnicas podem ser a solução. Vários podem ser os critérios para a personalização de um ambiente. Algumas pesquisas abordam personalização levando em consideração características demográficas, perfis, auto-regulação, cooperação, necessidades, entre outros. Para possibilitar um ambiente personalizado o conceito de percepção e contexto deve ser explorado. Contexto desempenha um papel importante em qualquer domínio que envolva requisitos como compreensão, raciocínio, resolução de problemas ou aprendizagem baseado em Santos et al. (2012).

Um sistema colaborativo sensível ao contexto é aquele que usa elementos de contexto das interações de um grupo para melhorar o desempenho do trabalho colaborativo, quer seja pelo provimento de informações mais apropriadas (filtragem seletiva e recomendação), quer seja pela habilitação de serviços relevantes (adaptação e personalização). O uso de contexto em sistemas colaborativos apoia o contato e a comunicação entre os atores, a compreensão mútua sobre o que ocorre dentro do grupo e o compartilhamento de conhecimento.

Outro tópico bastante discutido nas pesquisas é a utilização dos fóruns em ambientes MOOC. Segundo Nylén et al. (2015), as interações capturadas em fóruns de discussão fornecem uma visão valiosa sobre o processo de aprendizagem do aluno, pois pode mostrar como eles se relacionam com o conteúdo, sua compreensão e como estão interagindo com seus pares. O objetivo dessas análises de uma forma geral é avaliar de que forma acontecem as interações, quais os alunos que são considerados ativos, de que 
forma eles podem influenciar na aprendizagem dos demais colegas. Outra forma de análise sobre os fóruns é se a avaliação dos pares pode interferir na qualidade de um post e de que forma isso interfere na aprendizagem dos alunos. São utilizados de uma forma geral diversas metodologias de análise, mas a que predomina é observação e experimentação. Outras perspectivas indicam a realização de estudos que conectem Open Courseware e MOOCs, Learning Analytics em MOOCs, Cloud Computing e Mobile Apps para MOOCs considerando a diversidade do público-alvo, também requerem investigação pelos pesquisadores da área.

Um ramo de pesquisa que também tem chamado atenção, apesar das poucas pesquisas encontradas a respeito, é a inteligência emocional que, segundo Gil et al. (2015), descreve a capacidade de reconhecer e avaliar os seus próprios sentimentos e os dos outros, assim como a capacidade de lidar com eles. A proposta é melhorar a experiência do usuário coletando dados das redes sociais e/ou rastreando acessos para avaliar engajamento como forma de integrar e compartilhar aplicativos que possam melhorar a experiência do usuário.

A web social é um tópico muito discutido e estudado. Ao possibilitar o desenvolvimento de aplicações mais interativas, interessantes e eficientes, a Web 2.0 tornou-se um fator decisivo para o surgimento de uma World Wide Web que interliga pessoas, organizações e conceitos em vez de documentos, páginas ou recursos: a chamada Web Social, de acordo com Núñez et al. (2014). Desta forma, as pesquisas têm analisado que a utilização de ferramentas web sociais aumentam o nível de colaboração e engajamento entre os alunos de cursos online. Apesar dos resultados, pode-se perceber que as mídias são utilizadas de forma externa ao ambiente.

\section{Q4. De que forma as ações colaborativas são implementadas em ambientes MOOCs?}

Analisando as diversas ferramentas e ambientes colaborativos que são utilizados em plataformas MOOCs, existem diversas formas de abordagens que foram utilizadas visando garantir colaboração. Vários estudos têm sido realizados utilizando tutores inteligentes para garantir ambientes personalizados. Um sistema tutor de inteligência é aquele que faz a intermediação entre o aluno que busca um aprendizado e uma ferramenta facilitadora do aprendizado como dito por Núñez et al. (2014).

Outra forma de proporcionar personalização é através de sistemas de recomendação. Na maioria das pesquisas o ambiente é personalizado através de mineração de dados. Os dados filtrados geralmente estão relacionados com perfis, necessidades, hábitos. Sistemas de recomendação são uma sub-área de aprendizagem de máquina (machine learning) e tem por objetivo sugerir itens a um usuário, com base em seu histórico de preferências, de acordo com Zheng et al. (2016). As pesquisas geralmente utilizam a filtragem baseada em conteúdo, tentando direcionar os alunos baseados nos conteúdos de maior interesse dos alunos.

A gamificação também tem sido levantada como uma possibilidade de colaboração em MOOCS. Ao analisar a gamificação e MOOCs tem de ser levado em conta a "gamificação social" que cria relações mais estreitas entre plataformas e utilizadores, com o objetivo de promover o comportamento viral entre eles para aumentar a popularidade da plataforma. As características deste tipo de gamificação estão intimamente ligadas às características das redes sociais, baseado em Allal-Chérif et al. (2016). 
Outra forma de implementação bastante utilizada é a formação de grupos. Em um método de aprendizagem colaborativa, os critérios para a formação dos grupos influenciam os resultados. Os critérios incluem a quantidade de participantes nos grupos e o perfil dos participantes em cada grupo: grau de interesse do sujeito no assunto, conhecimento prévio, competência, nível de habilidade, maturidade, sexo, nacionalidade, dentre outras características dos participantes. Para Léris et al. (2016), a partir do perfil dos participantes, pode-se escolher entre a estratégia de homogeneidade ou de heterogeneidade.

\section{Considerações Finais e Trabalhos Futuros}

Os resultados obtidos a partir da RSL realizada mostram que é uma área em expansão e tem várias perspectivas de investigações futuras. Dentre elas podem ser destacadas a possibilidade de aplicação de técnicas de mineração de dados, Learning Analytics, gamificação, sistemas de recomendação, entre outros que foram citados nos artigos para disponibilizar ambientes personalizados aos alunos. Também merece ser comentado a utilização da web social como forma de integrar o contexto social nesses ambientes e disponibilizar a possibilidade de "juntar" pessoas com interesses comuns que possam compartilhar conteúdos e experiências garantindo a aprendizagem compartilhada. É evidente que para proporcionar a colaboração no ambiente MOOC é necessário integrar várias técnicas e ferramentas para proporcionar ambientes mais interativos que promovam o engajamento e motivação dos alunos levando em consideração o contexto dos alunos.

Como trabalhos futuros, pretende-se analisar se a disponibilização de ambientes colaborativos, através de Learning Analitycs, integrando contexto e recomendação de pessoas vai gerar ambientes mais colaborativos e interativos através de um experimento para que os dados possam ser validados.

\section{REFERÊNCIAS}

Allal-Chérif, Oihab; Bidan, Marc. Collaborative open training with serious games: Relations, culture, knowledge, innovation, and desire. Journal of Innovation \& Knowledge. 2016

Blanco, A. F., García-Peñalvo, F. J. and Sein-Echaluce, M. 2013.A methodology proposal for developing adaptive cMOOC", In: Proceedings of the First International Conference on Technological Ecosystem for Enhancing Multiculturality (TEEM '13), ACM, New York, NY, USA, 553-558.

Chauhan, Jyoti; Taneja, Shilpi; Goel, Anita. Enhancing MOOC with Augmented Reality, Adaptive Learning and Gamification. Published in: MOOCs, Innovation and Technology in Education (MITE), 3rd International Conference on. 2015.IEEE.

Coetzee, Derrick; Fox, Armando; Hearst, Marti A.; Hartmann, Bjorn. Chatrooms in MOOCs: All Talk and No Action. L@S '14 Proceedings of the first ACM conference on Learning @ scale conference. 2014. ACM

Dasarathy, Balakrishnan; Sullivan, Kevin; Schmidt, Douglas C.; Fisher, Douglas H.; Porter, Adam. The Past, Present, and Future of MOOCs and Their Relevance to 
VI Congresso Brasileiro de Informática na Educação (CBIE 2017)

Anais do XXVIII Simpósio Brasileiro de Informática na Educação (SBIE 2017)

Software Engineering. In: FOSE Proceedings of the on Future of Software Engineering. 2014. ACM.

Gené, Oriol Borrás; Nunes, Margarita Martínez; Fidalgo, Ángel. Gamification in MOOC: Challenges, Opportunities and Proposals for Advancing MOOC Model. TEEM '14 Proceedings of the Second International Conference on Technological Ecosystems for Enhancing Multiculturality. 2014. ACM.

Gil, Rosa; Virgili-Gomá, Jordi; García, Roberto; Mason, Cindy. Emotions ontology for collaborative modelling and learning of emotional Responses. Emotions ontology for collaborative modelling and learning of emotional responses.2015. Elsevier.

Kitchenham, B. Procedures for Performing Systematic Reviews. Joint Technical Report, TR/SE-0401 and NICTA 0400011T.1, Keele University. 2004. Disponível em http://www.idi.ntnu.no/emner/empse/papers/kitchenham_2004.pdf

Lerís, Dolores; Sein-Echaluce; Hernandez, Miguel; Bueno, Concepción. Validation of indicators for implementing an adaptive platform for MOOCs. Published in: Journal Computers in Human Behavior. 2016. SCIENCE.

Nelimarkka, Matti; Vihavainen, Arto. Alumni \& Tenured Participants in MOOCs: Analysis of Two Years of MOOC Discussion Channel Activity. L@S '15 Proceedings of the Second (2015) ACM Conference on Learning @ Scale. 2015. ACM.

Nylén, Aletta; Thota, Neena; Kinnunen, Paivi; Butler, Matthew; Morgan, Michael. Multidimensional analysis of Creative Coding MOOC forums - a methodological discussion. Koli Calling '15 Proceedings of the 15th Koli Calling Conference on Computing Education Research. 2015. ACM

Núñez, Margarita Martínez; Gené, Oriol Borrás; Blanco, Ángel Fidalgo. Social Community in MOOCs: Practical Implications and Outcomes. TEEM '14 Proceedings of the Second International Conference on Technological Ecosystems for Enhancing Multiculturality. 2014. ACM

Oliveira, Eduardo Araújo. i-collaboration 3.0: um framework de apoio ao desenvolvimento de Ambientes Distribuídos de Aprendizagem Sensíveis ao Contexto. Tese Doutorado. Universidade Federal de Pernambuco. Cin - Ciência da computação, 2013.

Santos, Vaninha Vieira; Tedesco, Patrícia; Salgado, Ana Carolina. Sistemas colaborativos para uma nova sociedade e um novo ser humano. $10^{\circ}$ capítulo do livro. Ed Campus. 2012.

Siemens, G. (2013) "Massive Open Online Courses: Innovation in Education?", eds. Commonwealth of learning, Perspectives on Open and Distance Learning: Open Educational Resources: Innovation, Research and Practice, p. 5. Disponível em: http://www.col.org/PublicationDocuments/pub_PS_OER-IRP_web.pdf. Acesso em dezembro 2016.

Zheng, Saijing; Wisniewski, Pamela; Rosson, Mary Beth; Carroll, John M. Ask the Instructors: Motivations and Challenges of Teaching Massive Open Online Courses. Proceeding CSCW '16. Proceedings of the 19th ACM Conference on ComputerSupported Cooperative Work \& Social Computing. Page 206-221. 2016. 